\title{
Estrogen Receptor and Progesterone Receptor Positive Pigmented Extramammary Paget's Disease of the Axilla Mimicking Melanoma: A Case Report and Review of the Literature
}

\author{
Chi Shyan Wang ${ }^{1}$, Hsuan Hsiang Chen ${ }^{2 *}$, Shih Hao Liu ${ }^{3}$ \\ ${ }^{1}$ Department of Dermatology, En Chu Kong Hospital, New Taipei City, Taiwan \\ ${ }^{2}$ Department of Dermatology, National Taiwan University Hospital and National Taiwan University College of Medicine, Taipei, Taiwan
}

${ }^{3}$ Department of Pathology, En Chu Kong Hospital, New Taipei City, Taiwan

Received: October 05, 2020; Accepted: October 12, 2020; Published: October 15, 2020

*Corresponding author: HsuanHsiang Chen, MD,Department of Dermatology, National Taiwan University Hospital, 7, Chung-Shan South Road,Taipei, Taiwan, Tel. No: +886-2-2356-2141; Fax. No:+886-2-2393-4177; E-mail: beauty101@gmail.com

\begin{abstract}
Pigmented extramammary Paget's disease (EMPD) is a rare variant which is often confused clinically and histologically with melanoma or other pigmented lesions. Herein, we describe a rare case of pigmented EMPD involving the axilla of a 40-year-old female thought initially to represent malignant melanoma clinically. Immunohistochemically, the neoplastic cells are positive for Estrogen Receptor (ER), Progesterone Receptor (PR), and weakly positive for Human Epidermal Growth Factor Receptor 2 (Her2). A review of the literature reveals that the pigmented variant of EMPD could be easily misdiagnosed as melanoma initially and additional confirmatory studies are almost always needed to confirm the diagnosis. The expression of ER and PR in pigmented EMPD is rarely mentioned previously. We believe it should be further investigated for the understanding of pathogenesis in pigmented EMPD and the potential role of adjuvant hormonal therapy.
\end{abstract}

Keywords: Extramammary Paget's Disease; Axilla; Estrogen Receptor; Progesterone Receptor

\section{Introduction}

Pigmented EMPD is an extremely rare neoplasm with only a few well-documented cases published so far [1-12]. Histologically, pigmented EMPD is characterized by intraepidermal Paget cells containing melanin pigment, surrounded by dendritic melanocytes. The relationship between Paget's cells and melanocytes remains unclear. Moreover, the expression of ER and PR in pigmented EMPD is rarely mentioned previously. The role of ER and PR in EMPD is still unclear and needs to be further explored.Herein, we describe a rare case of axillary pigmented EMPD with positive ER and PR staining immunohistochemically. To the best of our knowledge, this is the first reported case of pigmented EMPD on the axilla with positive ER and PR staining in the English literature.

\section{Case report}

A 40-year-old female presented to the clinic for excision of a $1.4 \times 1.4 \mathrm{~cm}$ dark brown pigmented irregular plaque (Figure 1)on the left axilla for 2 years. Clinically, the lesion was thought to represent a melanoma or dysplastic nevus. The patient had no clinical evidence of an underlying neoplasm on the axilla or either breast on palpation. There was no lymphadenopathy, and no genital lesion was noted. A left axillary skin excision was undertaken and the specimen measuring $2.2 \times 1.1 \mathrm{~cm}$ was entirely submitted for histopathological examination.

The histopathology of this biopsy was characterized

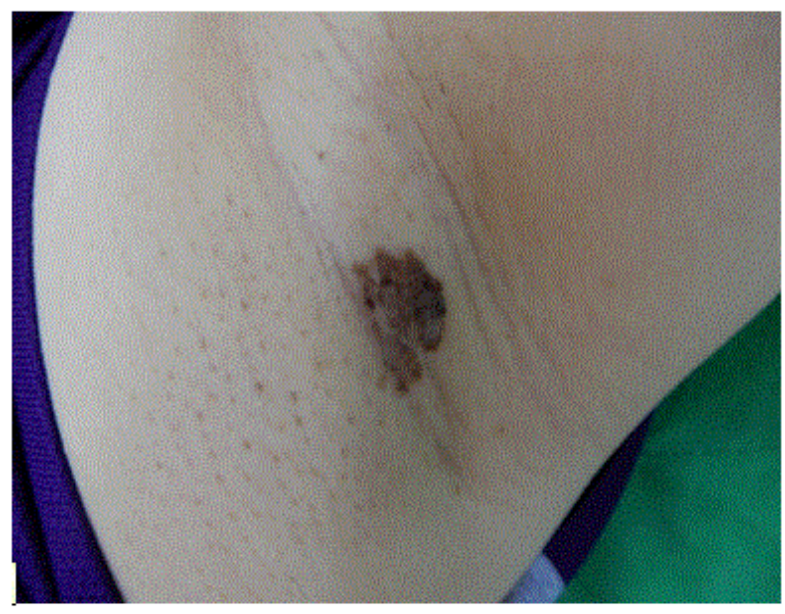

Figure 1: One 1.4x1.4 cm pigmented brownish plaque on the left axilla.

by clusters of neoplastic cells with pale cytoplasm, marked nuclear pleomorphism, hyperchromasia, and distinct nucleoli in the epidermis (Figure 2).There was no invasive carcinoma identified on multiple levels within the dermis or subcutis and the underlying apocrine sweat glands were normal. Immunohistochemically, the neoplastic cells are positive for ER, PR, and weakly positive for Her2 (Figure 3-5). Integrating the histological findings and immunohistochemical profile, the diagnosis of pigmented EMPD was made. The possibility of 


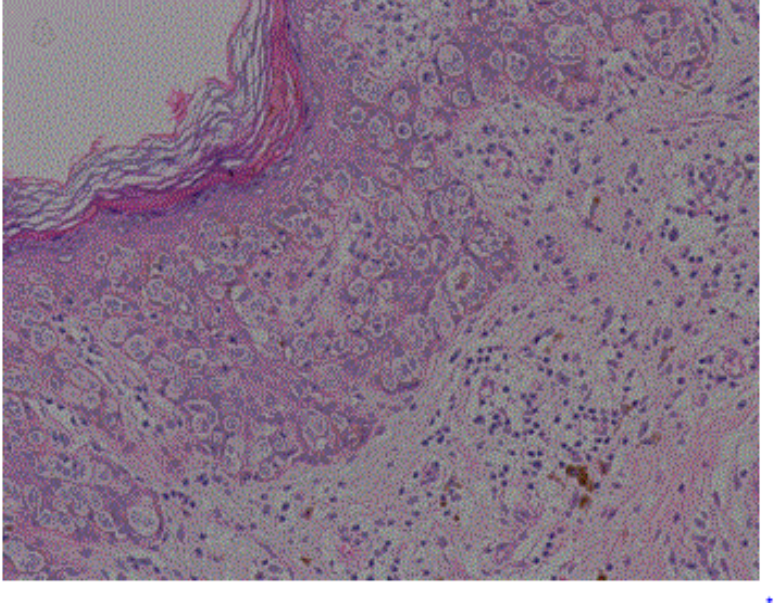

Figure 2: Pigmented EMPD showing dendritic melanocytes and malignant Paget cells with melanin pigment. Some melanophages were noted in the dermis resemblingthe regression of a melanoma $(\mathrm{H} \& \mathrm{E}$, original magnification $\times 200$ ) .

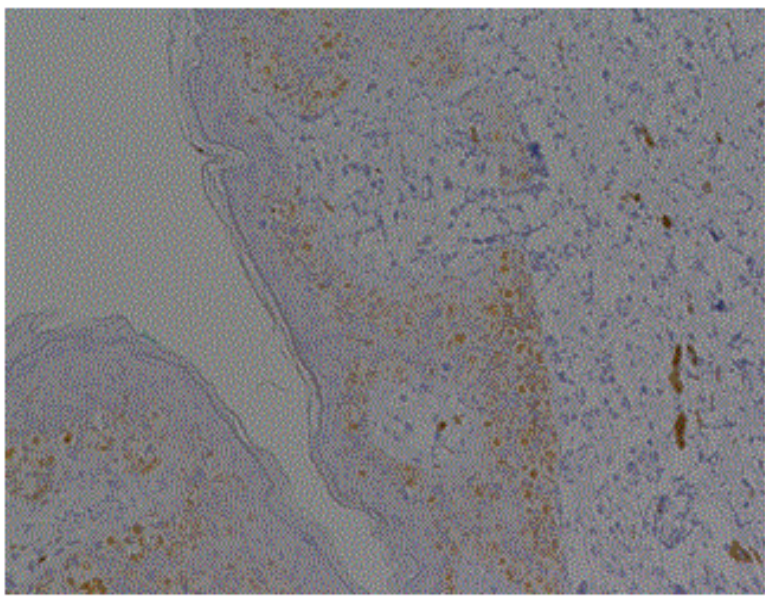

Figure 3: Immunohistochemical staining of ER (original magnification $\times 200$ ).

underlying breast cancer or malignancy of other sites cannot be excluded. Mammography of bilateral breasts revealed no discrete abnormal mass, stellate lesion, or clustered microcalcification. CT of the chest was obtained and showed no definite nodular lesion, air space consolidation, or atelectasis in bilateral lung fields. No definite mediastinal mass was found. The MRI of bilateral breasts showed only fibrocystic change. The laboratory examinations revealed normal thyroid function, CEA, and CA-153.Based on clinical and histopathological examination, this case most likely represents an example of primary axillary pigmented EMPD. No further treatment was given post excision because of negative section margins. However, the lesion recurred 16 months later after the primary excision.

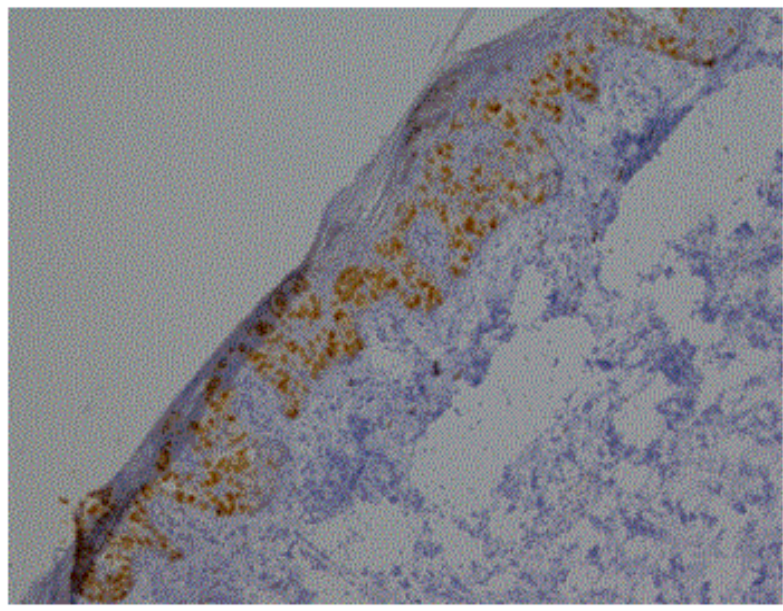

Figure 4: Immunohistochemical staining of PR (original magnification $\times 200)$.

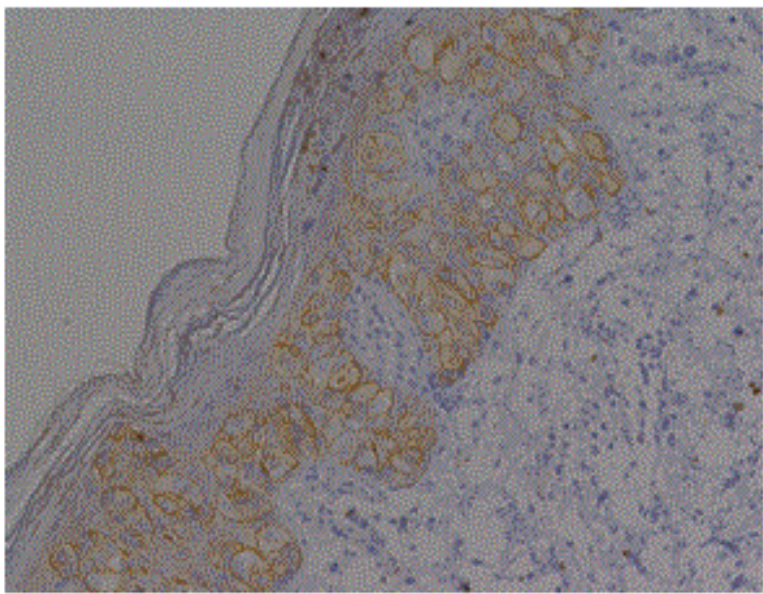

Figure 5: Immunohistochemical staining of Her2 (original magnification $\times 200$ ).

\section{Discussion}

EMPD is relatively uncommon with most cases occurring in the genital or perianal region[13].Axillary EMPD is rarely described in the literature. Simultaneous axillary and genital disease (also known as triple EMPD) were described exclusively in the Japanese literature[4].The cases of triple EMPD show a striking male predominance.One the other hand, isolated axillary involvement is more prevalent in females. The presence of an underlying carcinoma in axillary EMPD has been reported in $35 \%$ of the cases[4].A more recent study by Chiu et al. showed that only $14 \%$ of patients with unilateral axillary EMPD had an underlying carcinoma[14].

The pigmented variant of EMPD is an extremely rare entity with only a few well-documented cases previously reported in the literature (Table 1) [1-12]. The reported cases of pigmented EMPD occurred mostly in females with an average age of 63 years (range 43-83 years), and have involved mostly the vulvar or perineal region. Axillary involvement of pigmented EMPD 


\begin{tabular}{|c|c|c|c|c|c|c|c|c|}
\hline Study & Year & No & Age & Sex & Site & Cancer & $\begin{array}{l}\text { ER/PR } \\
\text { status }\end{array}$ & Ref \\
\hline Chiba et al. & 2000 & 2 & $\begin{array}{l}52 \mathrm{y} / \mathrm{o} \\
70 \mathrm{y} / \mathrm{o}\end{array}$ & $\begin{array}{l}F \\
F\end{array}$ & $\begin{array}{l}\text { Vulva } \\
\text { Vulva }\end{array}$ & $\begin{array}{c}\text { Breast } \\
-\end{array}$ & $\begin{array}{l}\text { N.A. } \\
\text { N.A. }\end{array}$ & 1 \\
\hline Gumurdula et al. & 2004 & 1 & $63 \mathrm{y} / \mathrm{o}$ & $\mathrm{F}$ & Perineum & - & N.A. & 2 \\
\hline Ko et al. & 2004 & 1 & $58 \mathrm{y} / \mathrm{o}$ & M & Scrotum & - & N.A. & 3 \\
\hline Hilliard et al. & 2009 & 1 & $79 \mathrm{y} / \mathrm{o}$ & M & Axilla & - & N.A. & 4 \\
\hline Petersson et al & 2009 & 1 & $43 \mathrm{y} / \mathrm{o}$ & $\mathrm{F}$ & Vulva & - & N.A. & 5 \\
\hline Vincent et al. & 2011 & 1 & $63 \mathrm{y} / \mathrm{o}$ & $\mathrm{F}$ & Abdomen & Colon & N.A. & 6 \\
\hline Lentini et al. & 2011 & 1 & $50 \mathrm{y} / \mathrm{o}$ & $\mathrm{F}$ & Vulva & - & N.A. & 7 \\
\hline Coras-Stepanek et al. & 2012 & 1 & $83 y / 0$ & M & Scrotum & - & N.A. & 8 \\
\hline Wang et al. & 2012 & 1 & $76 \mathrm{y} / \mathrm{o}$ & M & Axilla & Prostate & N.A. & 9 \\
\hline De la Garza Bravo et al. & 2014 & 1 & $51 \mathrm{y} / \mathrm{o}$ & $\mathrm{F}$ & Thigh & Breast & N.A. & 10 \\
\hline Ladak et al. & 2014 & 1 & $63 \mathrm{y} / \mathrm{o}$ & $\mathrm{F}$ & Axilla & - & - & 11 \\
\hline Kiavash et al. & 2019 & 1 & $74 \mathrm{y} / \mathrm{o}$ & $\mathrm{F}$ & Abdomen & - & N.A. & 12 \\
\hline Chen et al. & 2020 & 1 & $40 \mathrm{y} / \mathrm{o}$ & F & Axilla & - & + & $\begin{array}{c}\text { Current } \\
\text { case }\end{array}$ \\
\hline
\end{tabular}

N.A. : not available

was only found in 3 reported cases [4,9,11]. One previous report by Ohno et al. describes 2 cases of axillary EMPD which had a brownish appearance on clinical examination. However, there is no histologic description of pigmented Paget cells or an increased number of intraepidermal dendritic melanocytes [15].Our cases demonstrated intraepidermal Paget cells with abundant cytoplasm containing conspicuous melanin pigment. Besides, a reactive population of dendritic melanocytes was also noted in the epidermis, enveloping the tumor cells, which is similar to the case reported by Hilliard et al. [4].

The clinical relevance of this variant of EMPD resides in its potential to be misdiagnosed as malignant melanoma. The clinical differential diagnosis often includes anatypical melanocytic neoplasm or a pigmented Bowen's disease. The presenceof intraepidermal pagetoid cells containing cytoplasmic melanin pigment and the absence of anunderlying carcinoma may prompt the histological diagnosis of melanoma.The presence of dermal melanophages,inflammation, and fibrosis also mimick the regression of a dermal melanocytic component unless pigmented variant of EMPD is considered in the differential diagnosis. With the advent of immunohistochemical stainings, Paget cells usually stain positively for CK7, CK20, CAM 5.2, EMA, CEA, Her2/neu, BER-EP4, GCDFP15 (BRST2), or mucicarmine[7]. These cells are almost uniformly negative for melanocytic markers including S100, HMB45, MART1/Melan-A, and MITF.

The role of ER and PR in EMPD is still unclear. Zhou et al. reported the ER expression was 19.44\% in EMPD [16], which was higher than the previous rate in literature. Their data confirmed that the ER was rarely detected in EMPD.However, no significant difference in ER expression between EMPD(19.44\%) and MPD (9.09\%) was noted in their study. The expression of ER and PR in pigmented EMPD is not fully investigated.Ledak et al. reported a case of a 63-year-old female with axillary pigmented EMPD with positive Her2, but negative ER, and PR on immunohistochemical staining[11]. It is the only documented case describing the status of ER and PR in pigmented EMPD in previous English literature. Our case is the first reported case of pigmented EMPD on the axilla with positive ER and PR staining. We believe that the study of hormonal receptors might be a way to understand the pathogenesis of pigmented EMPD. The hormonal therapy, such as ER-inhibitor tamoxifen, may also be an alternative for selected ER-positive advanced cases.

The relationship between Paget's cells and melanocytes in pigmented EMPD remains unclear. It is hypothesized that the pigmentation could be the result of increased reactive melanocytes near the Paget's cells or the increased uptake of melanin from the surrounding melanocytes. The presence of reactive melanocytes within an epithelial neoplasm has been described in other cutaneous tumors, including melanoacanthomas[17,18], basal cell carcinomas [19],seborrheic keratosis, pigmented Bowen's disease [11],and melanocytic matricoma[20].On the other hand, the pigmentation of Paget cells could be the transfer of melanin from the surrounding melanocytes.It had been shown that Basic Fibroblastic Growth Factor (bFGF) and other chemotactic factors were the key factors in the transfer of pigment from the surrounding dendritic melanocytes[21,22].These cytokine factors may play the same role in pigmented EMPD as well.

Despite the recurrence rates for genital and perianal EMPD have been shown to be $32-50 \%$ and $50-70 \%$, respectively [23], a recent study of seven cases of unilateral axillary EMPD showed no recurrence after local resection [14].It is still not clear whether the recurrence rate of the pigmented variant of EMPD may be less than that of its non-pigmented variant. Because the lesion in our patient recurred 16 months later after the primary excision,we 
believe it is mandatory to follow up these patients regularly.

In summary, we described the unique case of pigmented EMPD in a female patient over the axillary region. We also intended to raise awareness of the potential pitfall of misdiagnosing pigmented EMPD both clinically and histologically as melanoma unless this variantof Paget's disease is considered in the differential diagnosis. The expression of ER and PR in pigmented EMPD should be further investigated for the understanding of pathogenesis in pigmented EMPD and the potential role of adjuvant hormonal therapy in this rare disease.

\section{References}

1. Chiba H, Kazama T, Takenouchi T, Nomoto S, Yamada S, et al. Two cases of vulval pigmented extramammary Paget's disease: histochemical and immunohistochemical studies. Br J Dermatol. 2000;142:1190-1194.

2. GumurdulaD, Sung CJ, Lawrence WD. Pathologic quiz case: a 63-yearold woman with a pigmented perineal lesion. Extramammary Paget disease. Arch Pathol Lab Med. 2004;128(1):e23-24.

3. Ko MJ, Hsiao CH, Tseng CJ, Liao YH. Pigmented extramammary Paget's disease. Dermatol Sin.2004;22:321-326.

4. Hilliard NJ, Huang C, Andea A. Pigmented extramammary Paget's disease of the axilla mimicking melanoma: case report and review of the literature. J CutanPathol. 2009;36(9):995-1000.

5. Petersson F, Ivan D, Kazakov DV, Kazakov DV, Michal M, et al Pigmented Paget disease-a diagnostic pitfall mimicking melanoma. Am J Dermatopathol. 2009;31(3):223-226.

6. Vincent J, Taube JM. Pigmented extramammary Paget disease of the abdomen: a potential mimicker of melanoma. Dermatol Online J. 2011;17(8):13.

7. Lentini M, Le Donne M. Asymmetrically pigmented patch on the vulvoperineal area: a quiz. Pigmented extramammary Paget's disease. ActaDermVenereol. 2011;91(3):380-381.

8. Stepanek B, Portatius A, Smith D, Stolz W. Dermatoscopy of pigmented extramammary Paget disease simulating melanoma. J Am AcadDermatol. 2012;67(4):e144-146.

9. Wang EC, Kwah YC, Tan WP, Lee JS, Tan Sh. Extramammary Paget disease: immunohistochemistry is critical to distinguish potential mimickers. Dermatol Online J. 2012;18(9):4.

10. Bravo MM, Curry JL, Cabala CA, Ivan DS, Drucker C, et al. Pigmented extramammary Paget disease of the thigh mimicking a melanocytic tumor: report of a case and review of the literature. J CutanPathol. 2014;41(6):529-535.

11. Ladak A, Bramley M, Titi S. Unilateral Pigmented Extramammary Paget's Disease of the Axilla Associated with a Benign Mole: A Case Study and a Review of Literature. Korean J Pathol. 2014;48(4):292 296.

12. Kiavash K, Kim S, Thompson AD. "Pigmented Extramammary Paget Disease"-A Potential Mimicker of Malignant Melanoma and a Pitfall in Diagnosis: A Case Report and Review of the Literature. Am J Dermatopathol. 2019;41(1):45-49.

13. Kanitakis J. Mammary and extramammary Paget's disease. J EurAcadDermatolVenereol. 2007;21:581-590.

14. Chiu CS, Yang CH, Chen CH. Extramammary Paget's disease of the unilateral axilla: a review of seven cases in a 20 -year experience. Int J
Dermatol. 2011;50(2):157-160.

15. Ohno H, Hatoko M, Kuwahara M, Ohnuma Y, Iida T, et al. Two cases of unilateral axillary Paget's disease. J Dermatol. 1998;25(4):260-263.

16.Zhou S, Zhong W, Mai R, Zhang G. Mammary and Extramammary Paget's Disease Presented Different Expression Pattern of Steroid Hormone Receptors. Biomed Res Int. 2017;2017:3768247.

17. Prince C, Mehregan AH, Hashimoto K, Plotnick H. Large melanoacanthomas: a report of five cases. J CutanPathol. 1984;11(4):309-317.

18. Schlappner OL, Rowden G, Philips TM, Rahim Z. Melanoacanthoma. Ultrastructural and immunological studies. J CutanPathol. 1978;5(3):127-141.

19. Florell SR, Zone JJ, Gerwels JW. Basal cell carcinomas are populated by melanocytes and Langerhans [correction of Langerhan's] cells. Am J Dermatopathol. 2001;23(1):24-28.

20. Williams CM, Bozner P, Oliveri CV, Horenstein MG. Melanocytic matricoma: case confirmation of a recently described entity. J CutanPathol. 2003;30(4):275-278.

21. Konomi K, Imayama S, Nagae S, Terasaka R, Chijiiwa K, Yashima Y. Melanocyte chemotactic factor produced by skin metastases of a breast carcinoma. J SurgOncol. 1992;50:62-66.

22. Saitoh K, Saga K, Okazaki M, Maeda K. Pigmented primary carcinoma of the breast: a clinical mimic of malignant melanoma. $\mathrm{Br} J$ Dermatol. 1998;139(2):287-290.

23. Zollo JD, Zeitouni NC. The Roswell Park Cancer Institute experience with extramammary Paget's disease. Br J Dermatol. 2000;142(1):5965. 